\title{
Apreciação corporal e modificação da aparência física em estudantes adolescentes de baixo poder aquisitivo
}

\author{
Ana Flávia Tavares \\ Angela Nogueira Neves Betanho Campana \\ Maria Sílvia de Moraes
}

\begin{abstract}
Resumo
O objetivo deste estudo foi caracterizar as técnicas e comportamentos de adolescentes de baixo poder aquisitivo para modificar a aparência física. A pesquisa é exploratória, transversal e utilizou-se de questionários estruturados. A amostra foi composta por 287 alunos do ensino médio, período diurno e noturno, de uma escola de região de baixo índice socioeconômico. Os resultados indicaram tendência à satisfação com o corpo, sendo a prática de atividade física regular o recurso mais frequente entre os rapazes e os regimes alimentares entre as moças para modificar a aparência. Foi encontrada uma diferença significante quanto à empregabilidade e à satisfação com o corpo, mas não em relação à estrutura da família. A população estudada não se revelou disposta a comprometer sua saúde para atender o padrão estético vigente.
\end{abstract}

Palavras-chave: Adolescente, aparência física, imagem corporal.

\section{Body appreciation and modification of physical appearance in poor class adolescents}

\begin{abstract}
In his work we aim at identifying techniques and behaviors used by poor adolescences to change their appearance. We developed an exploratory and transversal study by using structured questionnaires. The sample was composed by 287 high school students, from both periods (day and night) of a school located in a region of low socio-economic status. Results indicate a tendency to body satisfaction and to the practice of regular physical activity as the most frequent way of taking care of the body among boys. However, concerning to the girls, diets are most common technique to change their appearance. A significant difference regarding the body satisfaction and employability was found among those who study and work. We did not find any association regarding the structure of the family. The results indicate that the students in our study do not want to compromise their health in order achieve a "perfect body".
\end{abstract}

Keywords: Adolescents, physical appearance, body image.

\section{Evaluación corporal y modificación de la apariencia física en adolescentes de bajo poder adquisitivo}

\section{Resumen}

El objetivo de este estudio fue caracterizar las técnicas y comportamientos de adolescentes de bajo poder adquisitivo para modificar la apariencia física. La investigación é exploratoria, transversal y se valió de cuestionarios estructurados. La muestra se compuso por 287 alumnos de enseñanza media, períodos diurno y nocturno, de una escuela localizada en región de bajo índice socioeconómico. Los resultados señalaron tendencia a la satisfacción con el cuerpo además de indicar que los recursos más frecuentes para modificar la apariencia es la práctica de actividades físicas entre los chicos y las dietas alimentares entre las chicas. Se encontró una diferencia significante en cuanto a la empleabilidad y a la satisfacción con el cuerpo, pero no en relación a la estructura familiar. La población estudiada no se mostró dispuesta a poner su salud en riesgo para corresponder a los estándares estéticos vigentes.

Palabras Clave: Adolescente, apariencia física, imagen corporal. 


\section{Introdução}

O crescimento global e a disseminação de referenciais culturais - como, por exemplo, os relativos à produção artística, à estética, ao trabalho e aos meios de produção - contribuíram para um efeito globalizante, que pode ser notado em diferentes áreas de nossa vida (Moore, Chapman, \& Aiken, 2002). Dessa forma, a beleza nem sempre está nos olhos daquele um que vê, mas nos olhos da maioria. Ser considerado belo depende também da concordância com padrões de beleza vigentes, constituídos de acordo com parâmetros que determinam o adequado e o ideal naquela cultura específica (Mauss, 1974). Para as mulheres, belo é o corpo magro, a pele clara, cabelos lisos e olhos, de preferência, claros também (Grogan, 1999; Thompson, Heinberg, Altabe, \& Tantleff-Dunn, 1998; Vigarello, 2006). Para os homens, a beleza concretiza-se num corpo alto, musculoso e forte, com baixo percentual de gordura, ombros largos e rosto anguloso (Adams, Truner, \& Bucks, 2005; Ridegway \& Tylka, 2005; Schwartz \& Tylka, 2008).

Estar fora dessas expectativas pode gerar desde um estado de insatisfação até um profundo desconforto, que implica numa condição mais estressante e inibitória que a insatisfação corporal inicial. As conclusões negativas a respeito de si podem levar a comportamentos inibitórios ou autodestrutivos, com o propósito de eliminar a diferença que o exclui da norma e, por conseguinte, de seu grupo social (Corbin, Courtine, \& Vigarello, 2008; D’Andrea, 2003; Eco, 2007; Rosen, Orosan, \& Reiter, 1995).

Tanto homens quanto mulheres estão sujeitos a preocupações quanto às questões estéticas do seu corpo, sendo que são diferentes as atitudes em busca de atender o padrão de corpo ideal. Para as mulheres, foi descrito o drive to thinness, que se refere a uma forte vontade de ser magra. Entre os comportamentos relacionados ao drive to thinnness, encontram-se: prática de dietas restritas em calorias, uso de medicamentos para emagrecer - inibidores de apetite e hormônios da tireoide -, uso de laxantes, uso de roupas escuras e prática excessiva de atividade física (Anderson \& Bulik, 2004; Grogan, 1999; Thompson e cols., 1998). Em oposição a este construto, foi descrito recentemente para os homens o drive for muscularity. Refere-se ao desejo de alcançar um corpo muscular idealizado (Morrison, Morrison, \& McCann, 2006), ou ao grau de preocupação que o sujeito pode ter a respeito do aumento da sua musculatura (McCreay \& Sasse, 2000). Refere-se à necessidade de atender ao padrão de atratividade física masculina, no qual o sujeito mesomórfico, mais forte, mais musculoso e com menor percentual de gordura é tido como atraente (McCreary, Sasse, Saucier, \& Dorsch, 2004). Entre os comportamentos associados ao drive for muscularity encontram-se: uso de esteróides anabolizantes, prática de exercícios resistidos, uso de diuréticos, checagem constante do volume muscular e evitação do corpo (Pope, Pope, Menard, Olivardia, \& Phillips, 2005; Pope, Phillips, \& Olivardia, 2000; Olivardia, 2002).

$\mathrm{Na}$ adolescência, há uma série de fatores que podem contribuir para a acentuação das procupações com o corpo.
As alterações corporais causadas por ajustes fisiológicos próprios da puberdade podem resultar em distorção da percepção do corpo, desconforto corporal e, ainda, dores causadas pelo crescimento (Paxton, Eisenberg, \& Neumark-Sztainer, 2006). Essas alterações podem ser acentuadas e acompanhadas por um descontentamento acentuado com o corpo quando o adolescente avalia estar longe das normas do corpo ideal (Myers \& Crowther, 2009). Especificamente quanto às mudanças relativas ao peso e à constituição corporal, as evidências indicam que, apesar da magreza excessiva também ser fonte de insatisfação, o excesso de peso foi identificado como tendo maior influência na insatisfação corporal entre adolescente brasileiros de classe econômica baixa, média e alta (Pereira, Graup, Lopes, Borgatto, \& Daronco, 2009; Miranda, Conti, Bastos, \& Ferreira, 2011).

Durante o período de transição do corpo infantil para o corpo adulto, além das questões de ordem mais biológica - como crescimento, alterações hormonais, redistribuição do tecido adiposo no corpo, pele acneica -, os adolescentes têm a tarefa de integrar experiências afetivas, cognitivas e sociais com esse "novo corpo". As novas experiências no mundo exigem um potencial de resiliência para manter coesa sua identidade (Cyrulnik, 2004). Tal condição é favorecida por um ambiente familiar estável e por experiências corporais favoráveis, tendo o esporte e a educação física um papel importante nesse aspecto (Catusso, Campana, \& Tavares, 2010). O jovem com menos recursos para integrar experiências passadas e presentes, em especial aquelas de grande impacto emocional e/ou social, fica mais vulnerável aos extremos do drive to thinness e/ou drive for muscularity (D'Andrea, 2003; Tavares, 2003). Pesquisa prévia realizada com adolescentes de classes sociais desprivilegiadas na cidade de Vitória, Espírito Santo, revelou a presença de preocupação com as qualidades estéticas do corpo, além da preocupação com sua capacidade de trabalho - o corpo "aguentar" as exigências do dia a dia. Os quinze sujeitos entrevistados revelaram ter aspectos que gostariam de mudar em seu corpo e identificaram como corpos ideais para si próprios e para seus potenciais parceiros amorosos os corpos divulgados pela mídia. Ter menos recursos financeiros não foi fator limitador para não desejarem ou terem menor interesse no padrão de corpo midiático (Braga, Molina, \& Figueiredo, 2010).

Há uma identificação cada vez mais frequente de insatisfação com o corpo em diferentes faixas etárias, mas especialmente entre adolescentes (Ricciardelli \& McCabe, 2011; Wetheim \& Paxton, 2011). A forma como lidam com esta insatisfação e os comportamentos para modificarem seus corpos, por outro lado, são um assunto que ainda merece mais atenção por parte dos pesquisadores e daqueles profissionais que lidam diretamente com o público adolescente. Há de se considerar que o modo como se vestem, maquilam e o peso (massa corporal) que almejam quase sempre são estratégias adotadas a fim de se estabelecerem como membros de um grupo, se relacionar e ser aceito na sociedade: o corpo fora da norma do grupo é um corpo rejeitado (Enderle, 1988). Para pertencer ao grupo, compor- 
tamentos de risco como dietas bizarras, uso de laxantes, uso de esteroides anabolizantes, excesso de exercício, jejum, abuso de cirurgias plásticas estéticas - tanto em sua frequência quanto nas alterações provocadas - e uso de produtos químicos não apropriados para tingir os cabelos são exemplos de comportamentos que podem ter efeitos devastadores na saúde física e psicológica da pessoa (Jackson, 2004). Ao considerarmos que recursos financeiros escassos limitam o acesso aos profissionais de educação física e nutricionista (limitando seu acesso à prescrição e orientação de ações para emagrecimento e fortalecimento muscular dentro dos padrões saudáveis), ou a profissionais esteticistas (limitando os cuidados com a pele, as unhas, os cabelos com produtos e técnicas adequadas) e mesmo a cirurgiões plásticos, os profissionais que lidam com adolescentes de classes econômicas desprivilegiadas devem estar particularmente atentos ao agravamento dos efeitos da insatisfação com o corpo e ao uso de recursos para modificação da aparência (Huenemann, Shapiro, Hampton, \& Mitchell, 1966; Pastore, Fisher \& Friedman 1996; Thomas \& Sanandaraj, 1985).

Tendo esta argumentação em vista, o objetivo desta pesquisa foi aprofundar os conhecimentos do uso de recursos utlilizados para alcançar os padrões atuais de corpo ideal numa população brasileira adolescente, de ambos os sexos, de baixa renda. Procuramos também determinar se os jovens que compuseram nossa amostra usariam recursos que colocariam em risco sua saúde em busca do corpo ideal.

\section{Método}

Trata-se de um estudo transversal exploratório. Obedecendo à legislação de ética em pesquisa 196/96, esta pesquisa teve início apenas após sua aprovação pelo Comitê de Ética em Pesquisa (CEP-FAMERP). Todos os dados foram obtidos após a assinatura do Termo de Consentimento Livre e Esclarecido pelos voluntários e responsáveis.

\section{População e amostra}

A população alvo deste estudo foram jovens adolescentes, de ambos os sexos, de bairros de periferia que se enquadram nas classes sociais $C$ e $D$ de uma cidade do interior de São Paulo. A escolha dessa população deu-se por um motivo principal: buscavámos confirmar se, como proposto por Boltanski (2004), pessoas de classes populares no Brasil desejam alcançar os corpos midiáticos, direcionados para o consumo das classes mais enriquecidas. Os estudos anteriores no Brasil são qualitativos (Braga e cols., 2010) ou usaram amostras de classes sociais mistas (Pereira e cols., 2009), e uma ampliação destas informações, juntamente com a determinação dos recursos para a modificação da aparência, é importante. A seleção da amostra foi não probabilística, sendo a maior escola da periferia da cidade o centro de coleta de dados, e alunos de 11 salas de ensino médio, sendo 4 noturnas e 7 diurnas, foram convidados oralmente a participar da pesquisa.

Participaram da pesquisa 287 voluntários. A idade média da amostra foi 16,14 $\pm 1,12$ anos; altura 1,67 $\pm 0,09$

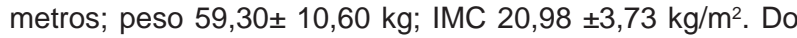
total da amostra, 45,67\% eram rapazes e 54,33\% eram moças, $65,5 \%$ têm uma estrutura familiar nuclear - moram com os pais e irmão(s). Ainda relativo à família, 98,6\% não têm filhos e 68,9\% se declararam solteiros. Quanto à ocupação $51,9 \%$ apenas estudam, 26,5\% estudam e trabalham sem carteira assinada e os demais estudam e trabalham com carteira assinada.

\section{Instrumentos}

- Questionário "Recursos à Alteração da Aparência": elaborado especialmente para esta pesquisa. O questionário continha itens como "Você mudaria algo no seu corpo para se sentir melhor?" ou "Em uma escala de zero (odeio meu corpo) a dez (adoro meu corpo), como você se sente em relação ao seu corpo?". As opções de resposta foram organizadas em escalas intervalares - de nunca (valor=1) a sempre (valor=6) - ou em escalas binomiais - não (valor =1) e sim (valor =2). Após ter sido concebido, o questionário foi submetido à analise de conteúdo por três profissionais da área com experiência no assunto. Os itens foram avaliados e apenas um item, que apresentou concordância quanto à sua adequacidade à pesquisa inferior a $80 \%$, foi eliminado. Estando a versão pré-final do intrumento de pesquisa pronta, realizou-se um pré-teste com cinco alunos do ensino médio de escola pública, os quais não apresentaram dificuldades no preenchimento.

- Questionário demográfico: também construído especificamente para esta pesquisa, destinou-se a coletar dados caracterizadores da amostra - como, por exemplo, a idade, peso, altura, etnia, constituição familiar. No pré-teste, também não foi identificada nenhuma dúvida quanto ao preenchimento deste questionário.

\section{Procedimentos para coleta de dados}

Inicialmente, obteve-se a prévia autorização da direção da escola para a realização da pesquisa. Em cada sala, no horário normal de aula, explicou-se a pesquisa e distribuiu-se o Termo de Consentimento Livre e Esclarecido (TCLE) destinado aos alunos a aos responsáveis. No dia seguinte, a pesquisadora voltou à escola e, após a assinatura dos alunos do TCLE e da permissão de participação da pesquisa dada pelos responsáveis dos menores, aplicou os questionários, que foram respondidos em sala de aula. Esse procedimento foi realizado em todas as salas de primeiro, segundo e terceiro anos do ensino médio da escola, nos períodos diurno e noturno. 


\section{Análise dos dados}

Usando o software SPSS, versão 15, buscou-se primeiramente identificar os dados outliers e perdidos. Em seguida, verificou-se a normalidade dos dados, empregando o teste de Kolmogorov-Smirnov. Foram empregados os testes de correlação de Spearman e os testes de variabilidade de Kruskal-Wallis e Mann-Whitney. Trabalhou-se com o intervalo de confiança de 95\%, sendo que nos testes de correlação considerou-se também resultados obtidos no intervalo de confiança de $99 \%$.

\section{Resultados}

Dos 294 alunos presentes nas salas de aulas visitadas da escola nos períodos de coleta, 287 concordaram em responder ao questionário. Nenhum dado outlier foi encontrado. Optou-se pela seleção listwise para evitar vieses nos dados. Dessa forma, sempre identificaremos quantos dados compuseram cada análise.

\section{Perfil da amostra quanto aos recursos para modificar a aparência}

Entre as adolescentes femininas, o uso de maquiagem e de outros produtos de beleza (descolorantes, perfumes, cremes para acne, loções autobronzeadoras) são os recursos utilizados com maior frequência. Não há uma grande tendência a fazer dietas restritivas em calorias, sendo que $70 \%$ da amostra feminina declarou não ter feito regime no último ano. Outra saída para o controle de peso seria a utilização de medicamentos para emagrecer; entretanto, nessa amostra, este não é um recurso muito ultilizado, tendo $93,8 \%$ da amostra afirmado nunca ter utilizado tais medicamentos. Da mesma forma, a prática de atividade física não constitui um recurso muito frequente para a amostra feminina, tendo apenas $28,6 \%$ declarado que faz atividade física 3 ou mais vezes por semana. A cirurgia plástica, definitivamente, não é um recurso para a modificação da aparência de que esta amostra feminina faz uso, com 99,3\% tendo declarado nunca ter sido submetida a um procedimento cirúrgico desta natureza.

Entre os rapazes, a atividade física e o uso de outros produtos cosméticos são os recursos mais frequentemente utilizados para o manejo da aparência. Praticam atividade física 3 ou mais vezes por semana $66,5 \%$ da amostra masculina e $49,6 \%$ usam produtos cosméticos para cuidar do corpo diariamente. Dietas restritivas, uso de medicamentos para emagrecer e submissão a cirurgias plástica não fazem parte do rol de recursos dessa amostra masculina (Tabela1).

A adoção de um medicamento que pudesse transformar a silhueta, o peso e a definição muscular destes jovens de acordo com seu ideal, mas de desconhecidos efeitos colaterais, também não se configura uma alternativa para a alteração da aparência, em nenhum dos dois sexos.

Identificaram-se algumas diferenças entre os adolescentes do sexo masculino e feminino que compuseram nossa amostra. Os rapazes praticam mais atividade física que as moças $\left(U=5189,00, N_{1}=135, N_{2}=150, p<0,001\right)$; as adolescentes fazem mais dietas restritivas $\left(\mathrm{U}=8348,00, \mathrm{~N}_{1}=\right.$ $\left.136, N_{2}=156, p<0,001\right)$ e com maior frequência $(U=8035,00$, $\left.N_{1}=136, N_{2}=156, p<0,001\right)$ que os adolescentes; os rapazes usam menos maquiagem $\left(\mathrm{U}=798,50, \mathrm{~N}_{1}=132, \mathrm{~N}_{2}=149\right.$, $\mathrm{p}<0,001)$ e produtos cosméticos $\left(\mathrm{U}=8081,50, \mathrm{~N}_{1}=135, \mathrm{~N}_{2}=\right.$ 148, $p=0,002$ ) que as moças.

O fator social relevante para esta amostra de baixa renda é a empregabilidade. Por isso, procuramos identificar diferenças entre os sujeitos da amostra que apenas estudam

Tabela 1. Uso de Recursos Para Modificação da Aparência.

\begin{tabular}{|l|l|l|l|}
\hline \multirow{2}{*}{} & \multicolumn{2}{|c|}{ Valores médios } \\
\cline { 2 - 4 } & Feminino & Masculino & $N$ total \\
\hline Frequência da Atividade física* & $2,73 \pm 1,62$ & $4,13 \pm 1,55$ & $3,38 \pm 1,73$ \\
\hline Adoção de Regimes restritivos em calorias** & $1,25 \pm 0,43$ & $1,05 \pm 0,22$ & $1,16 \pm 0,37$ \\
\hline $\begin{array}{l}\text { Frequência do uso de Medicamentos para } \\
\text { emagrecer* }\end{array}$ & $1,08 \pm 0,32$ & $1,10 \pm 0,56$ & $1,09 \pm 0,44$ \\
\hline Frequência do uso de Maquiagem* & $4,705 \pm 1,49$ & $1,22 \pm 0,85$ & $3,09 \pm 2,13$ \\
\hline Frequência do uso de outros produtos cosméticos* & $5,20 \pm 1,25$ & $4,48 \pm 1,77$ & $4,86 \pm 1,55$ \\
\hline Propensão a fazer Cirurgia plástica** & $1 \pm 0,08$ & $1,04 \pm 0,20$ & $1,02 \pm 0,15$ \\
\hline $\begin{array}{l}\text { Ingestão de medicamentos para melhora da } \\
\text { aparência com efeitos colaterais desconhecidos }\end{array}$ & $1,15 \pm 0,36$ & $1,11 \pm 0,32$ & $1,13 \pm 0,33$ \\
\hline
\end{tabular}

Nota: * para estas variáveis foi utilizada uma escala intervalar temporal, de 5 pontos, onde nunca $=1$ e sempre $=6$. * para estas variáveis foi utilizada um escala binomial, onde não $=1$ e sim $=2$. 
e que estudam e trabalham com e sem carteira assinada. 0 teste de Kruskall-Wallis indicou que aqueles que possuem carteira assinada praticam mais atividade física $\left(X^{2}=12,70\right.$, $d f=2, p=0,002)$ que os demais. Os sujeitos da amostra que apenas estudam fazem mais regimes $\left(X^{2}=7,210, \mathrm{df}=2\right.$, $p=0,027$ ) e com maior frequência que aqueles que trabaIham com e sem carteira assinada.

\section{Apreciação do corpo e o uso de recursos para modificar a aparência}

Os voluntários foram questionados quanto à nota que atribuiriam a seu corpo, numa escala de zero a dez, sendo o valor dez indicativo de que apreciavam bastante o corpo. A nota média da amostra foi $7,87 \pm 1,81$, havendo diferença significante entre a amostra feminina e masculina, tendo esta um nível maior de apreciação de seu corpo (U= 8276,00,
$\mathrm{N}_{1}=132, \mathrm{~N}_{2}=150, \mathrm{p}=0,015$; escores médios 7,65 $\pm 1,81$ e $8,14 \pm 1,77$ respectivamente). Aqueles que estudam e trabaIham com carteira assinada têm maiores índices de apreciação do corpo que os que apenas estudam ou que trabalham sem carteira assinada $\left(X^{2}=9,747, d f=2, p=0,008\right)$. Entre os jovens que vivem em uma estrutura familiar nuclear com estrututas "alternativas" - só com o pai, só com a mãe, só com avós ou tios, com madrasta, padrasto, amigos - não há nenhuma diferença significante quanto à apreciação do corpo.

Quando questionados se gostariam ou não de alterar alguma parte de seu corpo, identificamos uma diferença significante entre os sexos, sendo as adolescentes mais propensas a fazer mudanças que os rapazes $(U=6796,50$, $\mathrm{N}_{1}=135, \mathrm{~N}_{2}=151, \mathrm{p}<0,001$; escores médios 1,59 $\pm 0,49 \mathrm{e}$ $1,33 \pm 0,77$ respectivamente, sendo $2=s i m$ ). Também foi encontrada uma diferença significante quanto à empregabilidade $\left(X^{2}=9,997, \mathrm{df}=2, \mathrm{p}=0,007\right)$, sendo que aqueles que estudam e trabalham sem carteira assinada têm mais von-

Tabela 2. Correlações entre Apreciação Corporal e Técnicas de Alteração do Corpo, IMC e Idade para a Amostra Feminina (Diagonal Superior) e para a Amostra Masculina (Diagonal Inferior).

\begin{tabular}{|c|c|c|c|c|c|c|c|c|c|c|c|}
\hline & (1) & (2) & (3) & (4) & (5) & (6) & (7) & (8) & (9) & (10) & (11) \\
\hline (1) Gostar do Corpo & - & $-0,22^{\star \star}$ & 0,07 & $-0,18^{*}$ & $-0,24^{\star *}$ & $-0,26^{\star *}$ & $-0,05$ & $-0,05$ & 0,07 & $-0,22^{\star \star}$ & 0,10 \\
\hline $\begin{array}{l}\text { (2) Vontade de } \\
\text { mudar o corpo }\end{array}$ & $-0,17^{*}$ & - & $-0,12$ & 0,12 & $0,18^{*}$ & 0,04 & $0,19^{*}$ & 0,04 & 0,07 & 0,07 &,$- 014^{*}$ \\
\hline $\begin{array}{l}\text { (3) Frequência de } \\
\text { Atividade Física }\end{array}$ & $0,24^{\star *}$ & $-0,09$ & - & 0,05 & 0,13 & 0,30 ** & $-0,11$ & $-0,01$ & $-0,08$ & $0,27^{\star \star}$ & $-0,10$ \\
\hline $\begin{array}{l}\text { (4) Propensão a } \\
\text { adotar regimes } \\
\text { rigorosos }\end{array}$ & 0,03 & $0,31^{\star \star}$ & 0,09 & - & $0,83^{\star \star}$ & $0,19^{*}$ & $0,24^{\star \star}$ & $0,18^{*}$ & $0,15^{\star}$ & $0,36^{\star *}$ & $-0,05$ \\
\hline $\begin{array}{l}\text { (5) Freq. de regimes } \\
\text { rigorosos no último } \\
\text { ano }\end{array}$ & 0,02 & 0,09 & $-0,09$ & $0,70^{\star \star}$ & - & $0,25^{\star *}$ & $0,25^{\star \star}$ & $0,15^{\star}$ & $0,16^{*}$ & $0,42^{\star \star}$ & $-0,08$ \\
\hline $\begin{array}{l}\text { (6) Freq. de uso de } \\
\text { medicamentos para } \\
\text { emagrecer }\end{array}$ & 0,08 & $-0,05$ & $-0,15^{*}$ & 0,03 & $0,22^{\star \star}$ & - & 0,03 & $0,19^{*}$ & $0,32^{\star *}$ & 0,24 ** & 0,12 \\
\hline $\begin{array}{l}\text { (7) Frequência do } \\
\text { uso de maquiagem }\end{array}$ & $-0,02$ & 0,12 & $-0,13$ & 0,12 & 0,02 & $-0,05$ & - & $0,24^{\star \star}$ & 0,08 & 0,11 & 0,09 \\
\hline $\begin{array}{l}\text { (8) Freq. do uso de } \\
\text { outros produtos de } \\
\text { beleza }\end{array}$ & $-0,03$ & $0,43^{\star \star}$ & 0,03 & 0,03 & 0,02 & $-0,05$ & 0,07 & - & 0,06 & $-0,02$ & $-0,10$ \\
\hline $\begin{array}{l}\text { (9) Propensão } \\
\text { a fazer cirurgia } \\
\text { plástica }\end{array}$ & $-0,002$ & $-0,09$ & 0,11 & $-0,05$ & $-0,05$ & 0,04 & $-0,06$ & $-0,01$ & - & $-0,11$ & 0,06 \\
\hline $\begin{array}{l}\text { (10) Índice de } \\
\text { Massa Corporal } \\
\text { (IMC) }\end{array}$ & 0,03 & 0,03 & 0,05 & $0,40^{\star *}$ & $0,37^{\star \star}$ & $-0,06$ & $-0,08$ & $-0,15$ & $-0,10$ & - & $-0,10$ \\
\hline (11) Idade & 0,14 & 0,07 & 0,12 & $-0,08$ & $-0,12$ & 0,09 & $-0,07$ & 0,03 & 0,07 & $0,19^{*}$ & - \\
\hline
\end{tabular}

${ }^{*} \mathrm{p}<0,05,{ }^{* *} \mathrm{p}<0,01$. 
tade de mudar o corpo que aqueles que apenas estudam e estes mais vontade de mudar o corpo que aqueles estudam e trabalham com carteira assinada. A estrutura familiar não tem nenhum efeito na vontade de mudar o corpo.

Entre as adolescentes, maior apreciação do corpo associou-se significantemente ao menor índice de massa corporal, à menor vontade de fazer mudanças no corpo, ao menor uso de medicamentos para emagrecer e à menor adoção de dietas restritivas em calorias, inclusive no último ano. A maior vontade de mudar o corpo associa-se fracamente, porém com significância estatística, ao maior uso de maquiagem e ao maior número de dietas restritivas em calorias no último ano. Ressalta-se a associação positiva e moderada entre a maior frequência de atividade física e o maior uso de medicamentos para emagrecer. Entre os adolescentes, maior apreciação corporal associou-se à maior frequência de atividade física e menor vontade de mudar o corpo. A maior vontade de mudar o corpo, por sua vez, associa-se com significância estatística à maior adoção de regimes rigorosos e ao maior uso de outros produtos de beleza - como, por exemplo, hidratantes, condicionadores para o cabelo e bronzeadores. Para os adolescentes, também há a correlação positiva e significante entre a frequência de atividade física e o uso de remédios para emagrecer, sendo esta, porém, uma fraca associação (Tabela 2).

\section{Discussão}

O ideal de beleza e a expectativa quanto à aparência apresenta representações diferentes conforme o gênero em vários aspectos (Jackson, 2004; Tiggermann, 2004). Por isso, optamos em tabular os dados separando por sexo. Isso nos possibilitou reconhecer que o uso de atividade física como recurso para modificação da aparência é preponderante entre os homens. E, apesar do uso de dietas restritivas em calorias não ser o recurso mais usado entre as moças, elas o fazem com maior frequência que os rapazes. Os achados dessa pesquisa refletem os ideais encontrados na mídia, principalmente revistas de moda e de relacionamentos, nas quais os homens "modelos" possuem definição muscular nítida, corpos musculosos; as mulheres, por sua vez, são valorizadas pela sua magreza. Além disso, confirmam, numa amostra brasileira de baixa renda, o que vem sendo encontrado no cenário internacional (Thomsen, Weber, \& Brown, 2002).

Há uma maior apreciação corporal encontrada entre os rapazes que compuseram a amostra deste estudo, quando comparados às moças. Esse dado vem ao encontro de pesquisas anteriores - como, por exemplo, as de Avalos, Tylka e Wood-Barcalow (2005), Striegel-Moore e Franko (1990). A desfavorável condição socioeconômica não demonstrou ser um fator discriminante para a internalização de padrões de beleza, que incidem mais fortemente sobre as mulheres (Vigarello, 2006).

Entre as adolescentes, as correlações encontradas neste trabalho nos permitem afirmar que, quanto mais es- tas moças apreciam seu corpo, menor é a sua vontade de modificar seu corpo, menor o uso de medicamentos para emagrecer e a quantidade de dietas realizadas num intervalo de um ano. Conforme esperado, baixo índice de massa corporal associa-se a maior apreciação corporal. Os resultados também mostraram que, entre os adolescentes, aqueles que praticam mais atividade física apreciam mais seu corpo. Também, quanto mais apreciam seu corpo, menor é a vontade que tem de fazer alterações nele. Nas duas amostras há uma "coincidência" de fatores associados à apreciação do corpo: todos eles, de alguma forma, incidem sobre o manejo das formas corporais. Como nossa amostra se mostrou responsiva aos padrões de beleza vigentes veiculados pela mídia, este canal poderia também ser utilizado, com propagandas direcionadas a este público, para a aceitação do corpo real - a exemplo da "campanha da real beleza" veiculada por uma multinacional - com o propósito de fornecer informações e orientações corretas a respeito das diversas técnicas e produtos disponíveis no mercado, podendo favorecer a prevenção de "agressões" inadvertidas à saúde do adolescente de ambos os sexos.

Destaca-se um fator socioeconômico, a empregabilidade, provocando efeitos na apreciação corporal, na vontade de mudar o corpo e no uso de recursos para a modificação da aparência. Para os jovens que estudam e trabalham ter a carteira assinada parece ser um efeito protetor na constituição da sua identidade e de sua inserção no mundo social, diminuindo a possibilidade de viver as angústias desse período dirigindo sua agressividade ao corpo (Teles, 2001). Inesperada foi a ausência de efeito na constituição familiar na apreciação do corpo, sendo a família um referencial importante ao jovem. Podemos, entretanto, especular que esta geração, nascida já numa dinâmica de configurações alternativas de família, sofra menos o impacto de separações e da convivência de outras pessoas na intimidade, que não os pais e/ou irmãos, que as gerações passadas. Mas apenas uma pesquisa futura, dirigida a este ponto poderá fornecer maiores esclarecimentos.

O uso de artifícios para modificar o próprio corpo não se mostrou preocupante em termos de saúde pública. Apesar do desejo de alcançarem o corpo ideal, os participantes da pesquisa mostraram-se pouco adeptos às técnicas para modificar o corpo em que se desconhece os efeitos colaterais e, portanto, podem ser potencialmente prejudiciais à saúde do usuário.

Entretanto, há de se dar atenção à associação positiva moderada entre atividade física e uso de medicamentos para emagracer na amostra feminina. As adolescentes recusam o uso explícito de um medicamento que coloque em risco sua saúde, porém associam diretamente estas duas técnicas de modificação do corpo, potencialmente perigosas à saúde, quando realizadas sem orientação adequada. Isso indica que maior atenção deve voltar-se para o uso de recursos reconhecidos por eles como inócuos - batons, tintas, colorantes e sprays de cabelo, atividade física, regimes alimentares restritivos em calorias, uso de reguladores de apetite -, mas que, eventualmente, dependendo das condições 
de uso, podem afetar o equilíbrio orgânico e psicológico da saúde. Mais uma vez, campanhas educacionais dirigidas especificamente ao público adolescente poderiam ser úteis e uma pesquisa futura que se aprofundasse nessa questão permitiria explorar esses pontos, especialmente a associação entre exercícios e medicamentos para controle do peso, que parece não ser encarada como de risco, especialmente pelas adolescentes da amostra.

Concluímos que os adolescentes parecem dispostos a prezar por sua saúde, mas a desinformação pode levá-los pelo caminho oposto ao que desejariam. O profissional da saúde, ao atuar junto ao adolescente fornecendo informações e orientações corretas, pode ser efetivo na prevenção de danos à saúde dos adolescentes. Nessa direção, programas desenvolvidos nas escolas, nos locais de trabalho e nas Unidades Básicas de Saúde devem ser reconhecidos como de importância para orientar essa população e evitar práticas para mudar a aparência agressivas à saúde.

\section{Referências}

Adams, G., Truner, H., \& Bucks, R. (2005). The experience of body dissatisfaction in men. Body Image, 2, 271-283

Anderson, C. B., \& Bulik, C. M. (2004). Gender differences in compensatory behaviors, weight and shape salience, and drive for thinness. Eating Behaviors, 5, 1-11

Avalos, L., Tylka, T. L., \& Wood-Barcalow, N. (2005). The Body Appreciation Scale: Development and psychometric evaluation. Body Image, 2, 285-297.

Braga, P. D, Molina, M. C. B, \& Figueiredo, T. A. M. (2010). Representações do corpo: com a palavra um grupo de adolescentes de classes populares. Ciência \& Saúde Coletiva, 15, 87-95

Boltanski, L. (2004). As classes sociais e o corpo. Rio de Janeiro: Graal.

Catusso, R. L., Campana, A. N. N. B., Tavares, M. C. G. C. F. (2010). A Resiliência e a Imagem Corporal de Adolescentes e Adultos com Mielomeningocele. HuRevista, 36, 37-45

Corbin, A, Courtine, J., \& Vigarello, G. (2008). História do corpo. Petrópolis, RJ: Vozes.

Cyrulnik, B. (2004). Os patinhos feios. São Paulo: Martins fontes.

D'Andrea, F. F. (2003). Desenvolvimento da personalidade. Rio de Janeiro: Bertrand Brasil.

Eco, H. (2007). A história de feiura. São Paulo: Record.

Enderle, C. (1998). Psicologia da Adolescência. Porto Alegre :Artes Médicas.
Grogan, S. (1999). Body Image: understanding body dissatisfaction in men, women and children. Nova lorque: Routledge.

Huenemann, R. L., Shapiro, L. R., Hampton, M. C., \& Mitchell, B. W. (1996) A longitudinal study of gross body composition and body conformation and their association with food and activity in a teenage population. American Journal of Clinical Nutrition, 18, 325-338.

Jackson, L. A. (2004). Physical attractiveness: a sociocultural perspective. Em T. F. Cash \& T. Pruzinsky, Body Image - a handbook of theory, research e clinical practice (pp. 13-21). Nova Iorque: The Guilford Press.

Mauss, M. (1974). As técnicas corporais. Sociologia e antropologia. São Paulo: EPU/EDUSP.

McCreary, D. R., \& Sasse, D. K. (2000). An Exploration of the Drive for Muscularity in adolescent Boys and Girls. Journal of American College Health, 48, 297-304.

McCreary, D. R., Sasse, D. K., Saucier, D. M., \& Dorsch, K. D. (2004). Measuring the Drive for Muscularity: Factorial Validity of the Drive for Muscularity Scale in Men and Women. Psychology of Men \& Masculinity, 5, 49-58

Meyers, T. A., \& Crowther, J. H. (2009). Social comparison as a predictor of body dissatisfaction: a meta-analytic review. Journal of Abnormal Psychology, 118, 683-698.

Miranda, V. P. N, Conti, M. A, Bastos, R., \& Ferreira, M. E. C. (2011). Insatisfação corporal em adolescentes brasileiros de municípios de pequeno porte de Minas Gerais. Jornal Brasileiro de Psiquiatria, 60, 190-197.

Moore, S, Chapman, S, \& Aiken, D. (2002). Sociology for AS level. Glasgow: Collins Educational.

Morrison, T. G., Morrison, M. A., \& McCann, L. (2006). Striving for bodily perfection? An overview of the drive for muscularity. Em M. V. Kindes, Body Image: new research (pp. 1-34). Nova Iorque: Nova science Publication.

Olivardia, R. (2004). Body Image and Muscularity. Em T. F Cash \& T. Pruzinsky, Body Image: a handbook of theory, research \& clinical practice (pp. 210-218). Nova lorque: Guilford Press.

Pastore, D. R., Fisher M., \& Friedman, S. B. (1996). Abnormalities in weight status, eating attitudes, and eating behaviors among urban high school students: correlations with self-esteem and anxiety. Journal of Adolescence Health, 18, 312-319.

Paxton, S. J., Einsenberg, M. E., \& Neumark-Sztainer, D. (2006). Prospective predictors of body dissatisfaction in adolescents boys and girls: a five year longitudinal study. Developmental Psychology, 42, 888-899.

Pereira, E. F., Graup, S., Lopes, A. S, Borgatto, A. F, \& Daronco, 
L. S. E. (2009). Percepção da imagem corporal de crianças e adolescentes com diferentes níveis socio-econômicos na cidade de Florianópolis, Santa Catarina, Brasil. Revista Brasileira de Saúde Materno Infantil , 9, 253-262.

Pope, H. G, Phillips, K. A., \& Olivardia, R. (2000). The Adonis Complex. Nova lorque: The Free Press.

Pope, C. G., Pope, H. G., Menard, W., Fay, C., Olivardia, R., \& Phillips, K. A. (2005). Clinical features of muscle dysmorphia among males with body dysmorphic disorder. Body Image, 2, 395-400.

Ricciardelli, L. A., \& McCabe, M. P. (2011). Body Image development in adolescent boys. Em T. F. Cash \& L. Smolak, Body Image: a handbook of science, practice and prevention (pp.85-92). Nova Iorque: Guilford Press.

Ridegway, R. T., \& Tylka, T. L. (2005). College Men's Perceptions of Ideal Body Composition and Shape. Psychology of Men \& Masculinity, 6, 209-220.

Rosen, J. C, Orosan, P., \& Reiter, J. (1995). Cognitive behavior Therapy form negative Body Image in Obese Women. Behavior Therapy, 26, 25-42.

Schwartz, J. P., \& Tylka, T. L. (2008). Exploring entitlement as a moderator and mediator of the relationship between masculine gender role conflict and men's body esteem. Psychology of Men \& Masculinity, 9, 67-81.

Striegel-Moore, R. H., \& Franko, D. L. (1990). Body Image issues among girls and women. Em T. F. Cash \& T. Pruzinsky, Body Images: development, deviance and change (pp. 183 - 191). Nova Iorque: The Guilford Press.
Tavares, M. C. G. C. F. T. (2003). Imagem corporal: conceito e desenvolvimento. Barueri, SP: Manole.

Teles, M. L. S. (2001). Psicodinâmica do Desenvolvimento Humano: Uma Introdução à Psicologia da Educação. Petrópolis, RJ: Vozes.

Tiggermann, M. (2004). Media Influences on Body Image Development. Em T. F. Cash \& T. Pruzinsky, Body Image: a handbook of theory, research, e clinical practice (pp.91-98). Nova Iorque: The Guilford Press.

Thomas, I., \& Sanandaraj, H. S. (1985). A factor analytical study on the antecedents of self-esteem. Psychological Studies, 30, 97-101.

Thompson, J. K., Heinberg, L., Altabe, M., \& Tantleff-Dunn, S. (1998). Exacting beauty: theory, assessment and treatment of body image disturbance. Washington: APA.

Thomsen, S. R., Weber, M. M., \& Brown, L.B. (2002). The relationship between beauty and fashion magazines and the use of pathogenic dieting methods among adolescent females. Adolescence, 145, 1-18.

Vigarello, G. (2005). A história da Beleza. Rio de Janeiro: Ediouro

Wheetheim, E. H., \& Paxton, S. J. (2011). Body Image development in adolescent girls. Em T. F. Cash \& L. Smolak, Body Image: a handbook of science, practice and prevention (pp.76-84). Nova Iorque: Guilford Press.

Recebido em: 26/09/2011

Reformulado em: 22/02/2012

Aprovado em:07/03/2012

\section{Sobre as autoras}

Ana Flávia Tavares (aninhat88@gmail.com)

Graduada em Medicina, Faculdade de Medicina de São José do Rio Preto, São José do Rio Preto, São Paulo, Brasil

Angela Nogueira Neves Betanho Campana (angelanneves@yahoo.com.br)

Doutora em Atividade Física, Adaptação e Saúde pela Faculdade de Educação Física, Universidade Estadual de Campinas

Maria Sílvia de Moraes (mmoraes@famerp.br)

Professora doutora do Departamento de Epidemiologia e Saúde Coletiva Faculdade de Medicina de São José do Rio Preto, São José do Rio Preto, São Paulo, Brasil

Apoio financeiro: programa BIC/FAMERP e pelo Conselho Nacional de Desenvolvimento Científico e Tecnologico - CNPq. Processo número 140174/2009-5. 